\title{
Physical properties of the jet in $0836+710$ revealed by its transversal structure
}

\author{
M. Perucho and A. P. Lobanov
}

\author{
Max-Planck-Institut für Radioastronomie, Auf dem Hügel 69, 53121 Bonn, Germany \\ e-mail: perucho@mpifr-bonn.mpg.de
}

Received 4 April 2007 / Accepted 26 April 2007

\begin{abstract}
Studying the internal structure of extragalactic jets is crucial for understanding their physics. The Japanese-led space VLBI project VSOP has presented an opportunity for such studies, by reaching baseline lengths of up to $36000 \mathrm{~km}$ and resolving structures down to an angular size of $\approx 0.3$ mas at $5 \mathrm{GHz}$. VSOP observations of the jet in $0836+710$ at 1.6 and $5 \mathrm{GHz}$ have enabled tracing of the radial structure of the flow on scales from 2 mas to 200 mas along the jet and determination of the wavelengths of individual oscillatory modes responsible for the formation of the structure observed. We apply linear stability analysis to identify the oscillatory modes with modes of Kelvin-Helmholtz instability that match the wavelengths of the structures observed. We find that the jet structure in $0836+710$ can be reproduced by the helical surface mode and a combination of the helical and elliptic body modes of KelvinHelmholtz instability. Our results indicate that the jet is substantially stratified and different modes of the instability grow inside the jet at different distances to the jet axis. The helical surface mode can be driven externally, and we discuss the implications of the driving frequency on the physics of the active nucleus in $0836+710$.
\end{abstract}

Key words. galaxies: individual: 0836+710 - galaxies: jets - galaxies: nuclei - galaxies: active - radio continuum: galaxies

\section{Introduction}

Resolving the transversal (or radial) structure in extragalactic jets is a crucial step in our understanding of the physics of these objects. On parsec scales, this has become feasible only recently, using space VLBI ${ }^{1}$ observations with $\mathrm{VSOP}^{2}$ (Lobanov $\&$ Zensus 2001). These observations revealed the presence of a double helical structure inside the jet of 3C 273, which can be attributed to a combination of helical and elliptic modes of Kelvin-Helmholtz (KH) instability. Numerical simulations further support this interpretation (Perucho et al. 2006). In this letter, we expand the scope of this investigation and use VSOP observations of the radio jet in the quasar S5 $0836+710$ at 1.6 and $5 \mathrm{GHz}$ to estimate basic physical properties of the relativistic flow, focusing specifically on the radial profiles of its velocity and density.

The luminous quasar S5 $0836+710$ at a redshift $z=2.16$ hosts a powerful radio jet extending up to kiloparsec scales (Hummel et al. 1992). VLBI monitoring of the source (Otterbein et al. 1998) has enabled estimates of the bulk Lorentz factor $\gamma_{\mathrm{j}}=12$ and the viewing angle $\theta_{\mathrm{j}}=3^{\circ}$ of the flow. Presence of instability developing in the jet is suggested by the kink structures observed on milliarcsecond scales with ground VLBI (Krichbaum et al. 1990).

In the VSOP image of $0836+710$ at $5 \mathrm{GHz}$, oscillations of the jet ridge line are observed (Lobanov et al. 1998), with wavelengths of 7.7 mas and 4.6 mas. These structures have been identified with the helical surface and elliptic surface modes of $\mathrm{KH}$ instability, yielding estimates of basic physical properties of

\footnotetext{
1 Very Long Baseline Interferometry.

2 VLBI Space Observatory Programme.
}

the flow: the Lorentz factor $\gamma_{\mathrm{j}} \sim 11$, Mach number $M_{\mathrm{j}} \sim 6$ and jet/ambient medium density ratio $\eta\left(\rho_{\mathrm{j}} / \rho_{\mathrm{a}}\right)=0.04$. High dynamic range $\mathrm{VLBA}^{3}$ observations of $0836+710$ at $1.6 \mathrm{GHz}$ show the presence of an oscillation at a wavelength as long as $\sim 100$ mas (Lobanov et al. 2006). This wavelength cannot be reconciled with the jet parameters determined from the two shorter wavelength oscillations, indicating that the flow may have a complex, stratified transversal structure in which emission at lower frequencies originates from outer layers of the flow.

Taking into account that the parameters given in Lobanov et al. (1998) were obtained under the assumption of a vortex sheet transversal structure of the jet, we explore the possibility that the jet is sheared, with the long wavelength corresponding to a surface mode growing in the outer layers, whereas the shorter wavelengths are identified with body modes developing in the inner radii of the jet. In our approach, we assume that the magnetic field is not dynamically important and that the structure of the jet is due to KH instability. Under these assumptions, the jet structure and evolution can be described within the framework of linear stability theory for cylindric outflows.

In Sect. 2, we describe the method used to solve the linear stability problem for cylindric relativistic flows and provide the respective solutions for the set of parameters given in Lobanov et al. (1998). This solutions are compared in Sect. 3 with the wavelengths observed in the jet of $0836+710$. Section 4 summarizes the main results of this work and puts them in the broader context of the physics of relativistic outflows.

\footnotetext{
${ }^{3}$ Very Long Baseline Array of National Radio Astronomy Observatory, USA.
} 

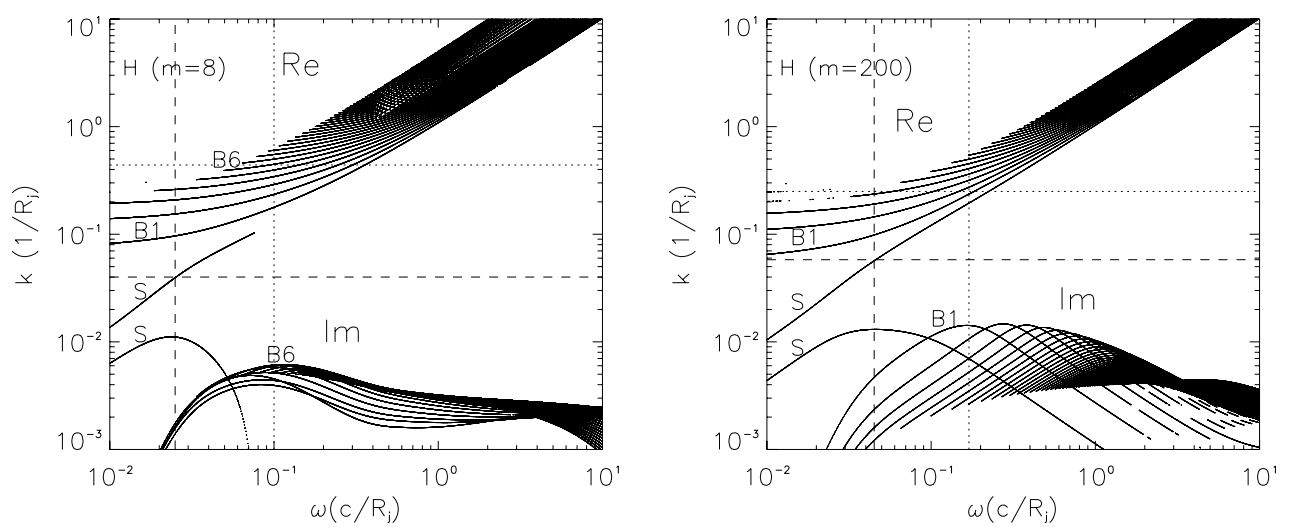

Fig. 1. Real (upper curves) and imaginary (lower curves) parts of the wavenumber versus frequency for the helical modes and the parameters given for the jet in $0836+710$. The vertical dashed lines indicate the minimum growth lengths of the surface modes, whereas the vertical dotted lines indicate the minimum growth length of the first body modes for $m=200$ and the minimum growth length of all body modes for $m=8$. The horizontal lines indicate the wave number at which the minimum growth length occurs. $S$ stands for the surface mode, $B 1$ for the first body mode, and $B 5-B 6$ indicate the body mode that gives the minimum growth lengths for $m=8$.

\section{Linear analysis}

We describe the flow in cylindrical coordinates $(r, z, \phi)$, with $r$ and $z$ defining the radial and axial directions, respectively. We consider a sheared transition in the axial velocity, $v_{z}$ and the rest mass density, $\rho$, between the jet and the ambient medium of the following form (see Perucho et al. 2007 for a detailed description of the linear analysis) $a(r)=a_{\infty}+\left(a_{0}-a_{\infty}\right) / \cosh \left(r^{m}\right)$, where $a(x)$ is the profiled quantity $\left(v_{z}\right.$ or $\rho$ ) and $a_{0}, a_{\infty}$ are its values at the jet symmetry plane (at $r=0$ ) and at $r \rightarrow \infty$, respectively. The integer $m$ controls the steepness of the shear layer. In the limit $m \rightarrow \infty$, the configuration turns into the vortex-sheet case, as described by Hardee (2000). The jet and the ambient medium are in pressure equilibrium. An adiabatic perturbation of the form $\propto g(r) \exp \left[i\left(k_{z} z-\omega t\right)\right]$ is introduced in the equations of the flow. Here, $\omega$ and $k_{z}$ are the frequency and longitudinal wavenumber of the perturbation, respectively, and the function $g(r)$ defines the radial structure of the perturbation. The resulting pressure perturbation $P_{1}$ is then obtained from a second order ordinary differential equation (Eq. (13) in Birkinshaw 1984). This equation can be solved by integrating from the jet axis to a point outside the jet, where boundary conditions on the amplitude of pressure perturbation and its first derivative are imposed. We apply the following boundary conditions: $a$ ) symmetry or antisymmetry of the perturbation on the jet axis; $b$ ) the Sommerfeld condition requiring the solutions to approach zero at infinity; and c) no incoming waves at infinity. These conditions ensure that the solutions can be given by Bessel functions inside the jet and Hankel functions outside. The solutions are obtained in the spatial domain, i.e., assuming $\omega$ is real and $k$ is complex. In this description, $1 / \mathcal{I}(k)$ gives the growth length (or $e$-folding length), defined as the distance at which the perturbation amplitude increases by one exponential factor. We adopt the jet parameters derived in Lobanov et al. $(1998,2006)$ and solve the pressure perturbation equation with several different values of $m$. The resulting solutions are plotted in Fig. 1 for the helical instability modes and for two values of $m: m=8$ (left panels) and $m=200$ (right panels). The $m=8$ case corresponds to a broad shear layer of $\sim 0.6 R_{\mathrm{j}}$ in width, whereas the $m=200$ one implies a shear layer width of $\sim 0.1 R_{\mathrm{j}}$, i.e., approaching the vortex-sheet case.

The plots in Fig. 1 indicate that, in thicker shear layers $(m=8)$, the surface mode grows faster than the body modes. The minimum growth lengths of the surface mode indicated by vertical dashed lines in Fig. 1 are realized at long wavelengths (horizontal dashed lines show the wavenumber of the mode): $\lambda_{\mathrm{S}, \mathrm{H}, 8}^{*} \sim 160 R_{\mathrm{j}}$. The smallest growth lengths of all body modes is achieved by the fifth and sixth order body modes at $\lambda_{\mathrm{B} 6,8} \sim 14 R_{\mathrm{j}}$, whereas the minimum growth length of the first body mode (expected to be among the most prominent in the jet) is realized at $\lambda_{\mathrm{B} 1,8} \sim 40 R_{\mathrm{j}}$.

For a thin shear layer $(m=200)$, the solutions approach the vortex-sheet approximation at the lowest frequencies (longest wavelengths). The minimum growth lengths of low order helical body modes are similar to that of the helical surface mode, with the minimum realized for the first body mode at $\lambda_{\mathrm{B} 1,200} \sim 25 R_{\mathrm{j}}$. The minimum growth length of the surface modes shifts to higher frequencies and shorter wavelengths $\left(\lambda_{\mathrm{S}, \mathrm{H}, 200}^{*} \sim 100 R_{\mathrm{j}}\right)$ compared to the respective values obtained for $m=8$. For $m<8$, the growths of all the modes are strongly reduced as the width of the shear layer is increased. For $m>200$ the solutions converge to the vortex-sheet case also at the highest frequencies/shortest wavelengths.

\section{Results}

The solutions of the linear stability problem obtained in the previous section can be compared with the wavelengths observed in the jet in $0836+710$. This comparison requires an estimate of the radius of the jet, in order to convert the frequencies and wavenumbers of the solution into physical units. The true jet radius can be estimated from the VLBA radio maps presented in Lobanov et al. (1998, 2006), using the following relation $R_{\mathrm{j}}$ (mas) $=0.5 \sqrt{D_{i}^{2}-b^{2}}$, where $D_{i}$ is the observed width of the jet and $b$ is the beam width transversally to the direction of the jet. We measure the diameter of the jet at $1 \%$ of the peak emission (Wehrle et al. 1992) at the base of the jet, implicitly assuming that the outer parts of the shear layer emit less than $1 \%$ of the radio power at these frequencies. This yields the jet radii of $R_{\mathrm{j}, 1.6} \sim 17$ mas and $R_{\mathrm{j}, 5} \sim 0.64$ mas at $1.6 \mathrm{GHz}$ and $5 \mathrm{GHz}$, respectively. With these values, the intrinsic (rest frame) wavelengths, $\lambda_{\text {int }}^{\text {mod }}$, can be obtained and expressed in units of $R_{\mathrm{j}}$. In order to compare these wavelengths with the observations, the observed wavelengths must be corrected for relativistic motion, projection and cosmological effects, yielding: $\lambda_{\text {int }}=\lambda_{\text {obs }}(1+z)\left(1-v_{\mathrm{w}} / c \cos \theta_{\mathrm{j}}\right) / \sin \theta_{\mathrm{j}}$, where $\lambda_{\mathrm{obs}}$ is the observed wavelength, $z$ is the redshift, $v_{\mathrm{w}}$ is the wave speed and $\theta_{\mathrm{j}}$ 
Table 1. Intrinsic wavelengths corresponding to the observed structures in the jet of $0836+710$.

\begin{tabular}{c|ccccc}
\hline \hline & $\begin{array}{c}\lambda_{\text {int }}(100 \text { mas }) \\
\left(v_{\mathrm{w}}=0.6 c\right)\end{array}$ & $\begin{array}{c}\lambda_{\text {int }}(100 \text { mas }) \\
\left(v_{\mathrm{w}}=0.8 c\right)\end{array}$ & $\begin{array}{c}\lambda_{\text {int }}(7.7 \text { mas }) \\
\left(v_{\mathrm{w}}=0.2 c\right)\end{array}$ & $\begin{array}{c}\lambda_{\text {int }}(7.7 \text { mas }) \\
\left(v_{\mathrm{w}}=0.5 c\right)\end{array}$ & $\begin{array}{c}\lambda_{\text {int }}(7.7 \mathrm{mas}) \\
\left(v_{\mathrm{w}}=0.7 c\right)\end{array}$ \\
\hline$R_{\mathrm{j}, 1.6}(17 \mathrm{mas})$ & $140 R_{\mathrm{j}}$ & $72 R_{\mathrm{j}}$ & $22 R_{\mathrm{j}}$ & $14 R_{\mathrm{j}}$ & $8 R_{\mathrm{j}}$ \\
$R_{\mathrm{j}, 5}(0.6 \mathrm{mas})$ & $4 \times 10^{3} R_{\mathrm{j}}$ & $2 \times 10^{3} R_{\mathrm{j}}$ & $620 R_{\mathrm{j}}$ & $390 R_{\mathrm{j}}$ & $230 R_{\mathrm{j}}$ \\
\hline
\end{tabular}

Notes: the wave speeds are given at the minimum growth length of the relevant modes: the helical surface mode for $m=8\left(v_{\mathrm{w}}=0.6 \mathrm{c}\right)$ and $m=200$ $\left(v_{\mathrm{w}}=0.8 c\right)$, the fifth and sixth body mode for $m=8\left(v_{\mathrm{w}}=0.2 c\right)$, and the first body mode for $m=8\left(v_{\mathrm{w}}=0.5 c\right)$ and $m=200\left(v_{\mathrm{w}}=0.7 c\right)$.

is the jet angle to the line of sight. The wave speed $v_{\mathrm{w}}=\omega / k$ is obtained from the solutions to the linear problem. The wave speed of the helical surface mode (most likely responsible for the longest observed wavelength of 100 mas) ranges from $v_{\mathrm{w}} \sim 0.6 \mathrm{c}$ to $v_{\mathrm{w}} \sim 0.8 c$, depending on the thickness of the shear layer. The dominant body modes (responsible for the shorter observed wavelengths of 7.7 mas and 4.6 mas) have speeds ranging from $v_{\mathrm{w}} \sim 0.2 c$ to $v_{\mathrm{w}} \sim 0.7 c$. Table 1 gives the intrinsic wavelengths for two main observed wavelengths (100 mas and 7.7 mas), calculated with different appropriate wave speeds in units of the jet radius. The respective intrinsic wavelengths of the 4.6 mas mode can be obtained by dividing the values for the 7.7 mas mode by a factor of 1.7 .

We note that the wavelengths obtained for the $m=200$ case cannot be reproduced by any combination of excited $\mathrm{KH}$ modes at, or close to, their resonant frequencies, although the lack of error estimations prevents us from completely ruling out this possibility. This fact alone points towards the jet being transversally stratified. Further evidence for this comes from a detailed analysis of the broad shear layer case $(m=8)$.

For the $m=8$ case and for $R_{\mathrm{j}, 5}$, the intrinsic wavelengths for the longest structure ( 100 mas) have no counterparts in the solutions to the linear problem, as already pointed out by Lobanov et al. (2006). This suggests that the radiating particles emitting at $5 \mathrm{GHz}$ occupy a central spine in a stratified jet, while the longer-wavelength structure seen at $1.6 \mathrm{GHz}$ is generated in outer layers of the flow.

The intrinsic wavelengths obtained for $R_{\mathrm{j}, 1.6}$ are close to the most unstable wavelengths provided by the $m=8$ shear layer. In this case, the helical 100 mas $\left(140 R_{\mathrm{j}}\right)$ structure, corresponding to a wavenumber $k \sim 0.045$, can be identified with $\lambda_{\mathrm{S}, \mathrm{H}}^{*} \sim 160 R_{\mathrm{j}}\left(k_{\mathrm{S}, \mathrm{H}}^{*} \sim 0.04\right)$. The helical 7.7 mas $\left(22 R_{\mathrm{j}}\right)$ structure can be identified with the fifth or sixth body mode excited at $\lambda>\lambda_{\mathrm{B} 6, \mathrm{H}}^{*} \sim 14 R_{\mathrm{j}}$, and the elliptic 4.6 mas $\left(13 R_{\mathrm{j}}\right)$ structure would correspond to the fifth and sixth elliptic body modes growing at $\lambda_{\mathrm{B} 6, \mathrm{E}}^{*} \sim 14 R_{\mathrm{j}}$, as indicated by the linear solution (not shown). We consider this a better explanation, and we adopt $R_{\mathrm{j}, 1.6}$ as the generic jet radius $R_{\mathrm{j}}$ in the following discussion.

One possible alternative to the stratified jet scenario is to consider deviations of the basic jet parameters from those derived in Lobanov et al. (1998, 2006). To assess this alternative, we have calculated the solutions of the stability equation for different sets of parameters, varying the jet Lorentz factor, restmass density ratio and specific internal energy. Our conclusions are summarized below: 1) we find that for colder jets (with a sound speed $c_{\mathrm{s}, \mathrm{j}} \sim 0.054$, corresponding to $M \sim 18$ ) the growth lengths $\left(k_{i}^{-1} \geq 10^{3} R_{\mathrm{j}}\right)$ of the surface and first body modes are too large to explain the growth of the instability in the jet. 2) For hotter jets $\left(c_{\mathrm{s}, \mathrm{j}} \sim 0.55, M \sim 1.8\right)$, no body modes exist that could reproduce the observed 7.7 mas and 4.6 mas wavelengths. At the same time, the surface modes alone cannot reproduce the three wavelengths observed. 3$)$ Lighter jets $\left(\eta=4 \times 10^{-4}\right)$ produce solutions similar to those arising for colder jets, with the exception that the growth lengths are slightly shorter in this case, but still of the order of $500 R_{\mathrm{j}}$, i.e., even longer than the longest observed wavelength taking the radius of the jet at $1.6 \mathrm{GHz}$. 4) Denser jets $(\eta=0.4)$ show the surface and first body modes at the appropriate wavelengths, but the surface mode grows at a too long growth length $\left(k_{i}^{-1} \sim 500 R_{\mathrm{j}}\right)$. Moreover, the observations indicate that the surface mode must grow faster than the body modes, contrary to the results derived for a dense jet. 5) Slower jets $\left(\gamma_{j}=2\right)$ show very short growth lengths for the surface mode $\left(k_{i}^{-1} \sim 17 R_{\mathrm{j}}\right)$ and the solutions yield too short wavelengths for the body modes. 6) Finally, much faster jets $\left(\gamma_{j}=100\right)$ produce too long growth lengths. This analysis indicates that the jet in $0836+710$ must have a Lorentz factor close to 12 or slightly smaller, a density ratio of $10^{-2}-10^{-1}$ and a sound speed $c_{\mathrm{s}, \mathrm{j}} \sim 0.2$ or slightly larger - not deviating much from the parameters derived in Lobanov et al. $(1998,2006)$.

\section{Discussion}

The description developed in this paper explains all three major oscillations observed in the jet of $0836+710$, if the flow speed and density are both transversely stratified. The longest observed wavelength of 100 mas represents the helical surface mode, whereas the two shorter ones $(7.7$ mas and 4.6 mas) are identified with the fifth/sixth order helical and elliptic body modes, respectively. It is also possible that the short wavelengths correspond to a lower order body mode (first body mode, for example) growing not at a resonant wavelength.

It has been shown by Hardee et al. (1994) that the helical surface mode of KH instability can be driven by an external periodic process. In this case, coupling this process to the helical surface mode requires the driving frequency to be lower than the resonant frequency. For $0836+710$, the driving frequency of the helical surface mode at its minimum growth length is $0.025 \mathrm{c} / R_{\mathrm{j}}$ would imply a driving period of $T_{\mathrm{dr}} \sim 5.6 \times 10^{7} \mathrm{yrs}$. This is similar to the driving period of $\sim 2 \times 10^{7}$ yrs found for 3C 449 (Hardee et al. 1994).

The period $T_{\mathrm{dr}}$ can be produced by a number of physical processes, including a binary black hole and a misaligned torus as the most plausible (Appl et al. 1996). The latter can however be discarded after Lu \& Zhou (2005) have shown that the absolute magnitude of the galaxy in the $B$ band anticorrelates with the precession periods due to misalignment between the black hole rotation axis and the outer regions of the accretion disk. The absolute magnitude of $0836+710$ in the $R$ band is $M_{R}=-29.9$ (Caccianiga et al. 2002). Assuming a similar magnitude for the $B$-band yields precession periods of $10^{3}-10^{5} \mathrm{yrs}$, depending on the accretion disk and black-hole properties (Lu $\&$ Zhou 2005). This is much shorter than $T_{\mathrm{dr}}$ we have derived. Combining $T_{\mathrm{dr}}$ with the estimate of the black hole mass in $0836+710\left(2 \times 10^{8}-10^{9} M_{\odot}\right.$; Tavecchio et al. 2000$)$ yields a companion mass of $10^{4}-10^{7} M_{\odot}$, depending on the separation between the two black holes $\left(10^{17}-10^{18} \mathrm{~cm}\right)$.

Also, the kiloparsec-scale structure of $0836+710$ shows a large, decollimated feature (Hummel et al. 1992), without any 
emission between it and the $1.6 \mathrm{GHz}$ jet. It remains to be tested by new high dynamic range observations whether the long instability mode ultimately causes the disruption of the flow, and produces this decollimation.

Acknowledgements. This work was supported in part by the Spanish Dirección General de Enseñanza Superior under grants AYA-2001-3490-C02 and AYA2004-08067-C03-01. M.P. is supported by a postdoctoral fellowship of the Generalitat Valenciana (Beca Postdoctoral d'Excel-lència).

\section{References}

Appl, S., Sol, H., \& Vicente, L. 1996, A\&A, 310, 419

Birkinshaw, M. 1984, MNRAS, 208, 887

Caccianiga, A., Marcha, M. J., Antón, S., Mack, K. H., \& Neeser, M. J. 2002, MNRAS, 329, 877
Hardee, P. E. 2000, ApJ, 533, 176

Hardee, P. E., Cooper, M. A., \& Clarke, D. A. 1994, ApJ, 424, 126

Krichbaum, T. P., Hummel, C. A., Quirrenbach, A., et al. 1990, A\&A, 230, 271

Lobanov, A. P., \& Zensus, J. A. 2001, Science, 294, 128

Lobanov, A. P., Krichbaum, T. P., Witzel, A., et al. 1998, A\&A, 340, 60

Lobanov, A. P., Krichbaum, T. P., Witzel, A., \& Zensus, J. A. 2006, PASJ, 58, 253

Lu, J. F., \& Zhou, B. Y. 2005, ApJL, 635, 17

Hummel, C. A., Muxlow, T. W. B., Krichbaum, T. P., et al. 1992, A\&A, 266, 93

Otterbein, K., Krichbaum, T. P., Kraus, A., et al. 1998, A\&A, 334, 489

Perucho, M., Lobanov, A. P., Martí, J. M., \& Hardee, P. E. 2006, A\&A, 456, 493

Perucho, M., Lobanov, A. P. 2006, Proceedings of Primer Encuentro de la Radioastronomía Española, ed. J. C. Guirado, I. Martí \& J. M. Marcaide, in press [arXiv: astro-ph/0607386]

Perucho, M., Hanasz, M., Martí, J. M., \& Miralles, J. A. 2007, Phys. Rev. E, submitted

Tavecchio, F., Maraschi, L., Ghisellini, G., et al. 2000, ApJ, 543, 535

Wehrle, A. E., Cohen, M. H., Unwin, S. C., et al. 1992, ApJ, 391, 589 